\title{
Human Body Digitalization and Marketing Consequences in the Coming Decades
}

\author{
Florin Popescu ${ }^{1}$, Cezar Scarlat ${ }^{1}$, Valentin $\operatorname{Stan}^{2}$ \\ ${ }^{1}$ Faculty of Entrepreneurship, Business Engineering and Management, University "Politehnica", Bucharest, Romania \\ ${ }^{2}$ Electronics and Remote Controls Applied in Transports Systems, University “Politehnica”, Bucharest, Romania
}

Email address:

popescuveve@gmail.com (F. Popescu), cezarscarlat@yahoo.com (C. Scarlat), valentin.stan@smart-tc.ro (V. Stan)

\section{To cite this article:}

Florin Popescu, Cezar Scarlat, Valentin Stan. Human Body Digitalization and Marketing Consequences in the Coming Decades. American Journal of Embedded Systems and Applications. Vol. 5, No. 3, 2017, pp. 21-28. doi: 10.11648/j.ajesa.20170503.11

Received: October 26, 2017; Accepted: November 30, 2017; Published: January 2, 2018

\begin{abstract}
Human body digitization, in other words "mating" with technology, represents a fusion of technologies that shrinks the boundaries of physical, digital and biological world. Throughout history, trade perception has changed significantly, from a mere intermediary to creative activities. Adapting marketing activities and strategy, generated by the technology development of modern society requires in-depth marketing research on client profile, customers' suggestions as well as their purchase capacity, consumption habits and other matters, which are at the ground of demand and trade policies. The authors consider that the technology incorporated in human body will crucially affect the marketing activities and strategy, during the coming decades. By human body digitalization, for example, marketers can have real-time information about the time clients spent in front of a product, various reactions they might have, their level of satisfaction, and which alternatives they are considering. Human body digitization process will crucially affect industrial companies, which will truly become digital businesses with basic physical products complemented by digital interfaces and innovative data services. These digital businesses will directly work with customers and suppliers in digital industrial ecosystems, which represent a radical change in the fundamentals of today's marketing. It is very likely for many companies that data analysis methods can have a significant influence on decision-making processes over the next decade. The authors conclude that the human body digitalization - by collecting various marketing data on client behavior - will dramatically influence the way of future marketing research, and will inherently lead to radical shifts in the marketing area in the coming decades. More than that, the authors of this futuristic study go ahead with forecasts, saying Human Body Digitization will lead to a complete globalization of the services and products market.
\end{abstract}

Keywords: Human Body Digitalization, Marketing Data Collection, Client Behavior

\section{Introduction}

For a long time, humans considered that humanity has achieved its evolutionary peak. Today those who try to foresee the future of humankind and its evolution are thinking of cyborg image, a combination of a biological creature and a technological masterpiece [1].

Electronics world is progressing at a very fast rate and today there are many revolutionary ideas, projects already underway that will end up "tomorrow" or in a few years. The three authors, specialized in electronic field, predict that with the unprecedented development of electronics, there will be also a major change of paradigm in industrial business, as well as in the marketing arena. According to their vision, the major change in the marketing arena is that through the new electronic technology incorporated within human body, it will be possible not only to collect different biometric data from consumers in real time, right in the supermarkets, but also to identify the elements underlying the purchasing decision. Thus, after analyzing how customers react in the supermarket, marketers can modify product design, eliminating images or aspects, which do not produce an emotional reaction, and emphasizing those that produce emotional reactions from customers. It seems that this is the future, or at least the vision of the authors.

The study focuses on four directions of analysis: firstly, presents a short historical evolution of electronic world 
secondly is analysed the brave new world of chips and biometrics, thirdly subcutaneous devices - merger of biological and electronic world and eventually predicts and discuss the marketing consequences of imbedded technology.

\section{Method}

The trigger point of this study was the fact that last year, in 2016, in Davos, were discussed and anticipated the future drivers of changing the world of industry and, implicitly, marketing strategy. But, from the point of view of the authors of this futuristic study, world strategists have failed to consider the possible impact of human body digitalization process, as well as, its implications on industry and, implicitly, on marketing strategy. According to the authors futuristic vision, we cannot discuss about 4.0 Revolution, without taking into account the human body digitalization process, which is almost a reality, even though not accepted by everybody.

In below ranking realized by World Economic Forum, Davos, 2016, the authors recommend a reorganization of the positions of the drivers of changes, including in the top positions of this ranking the human body digitization process. This futuristic vision represents, from the point of view of the authors, a worthy perspective to take into consideration in setting the priorities of the World Economic Forums that will follow.

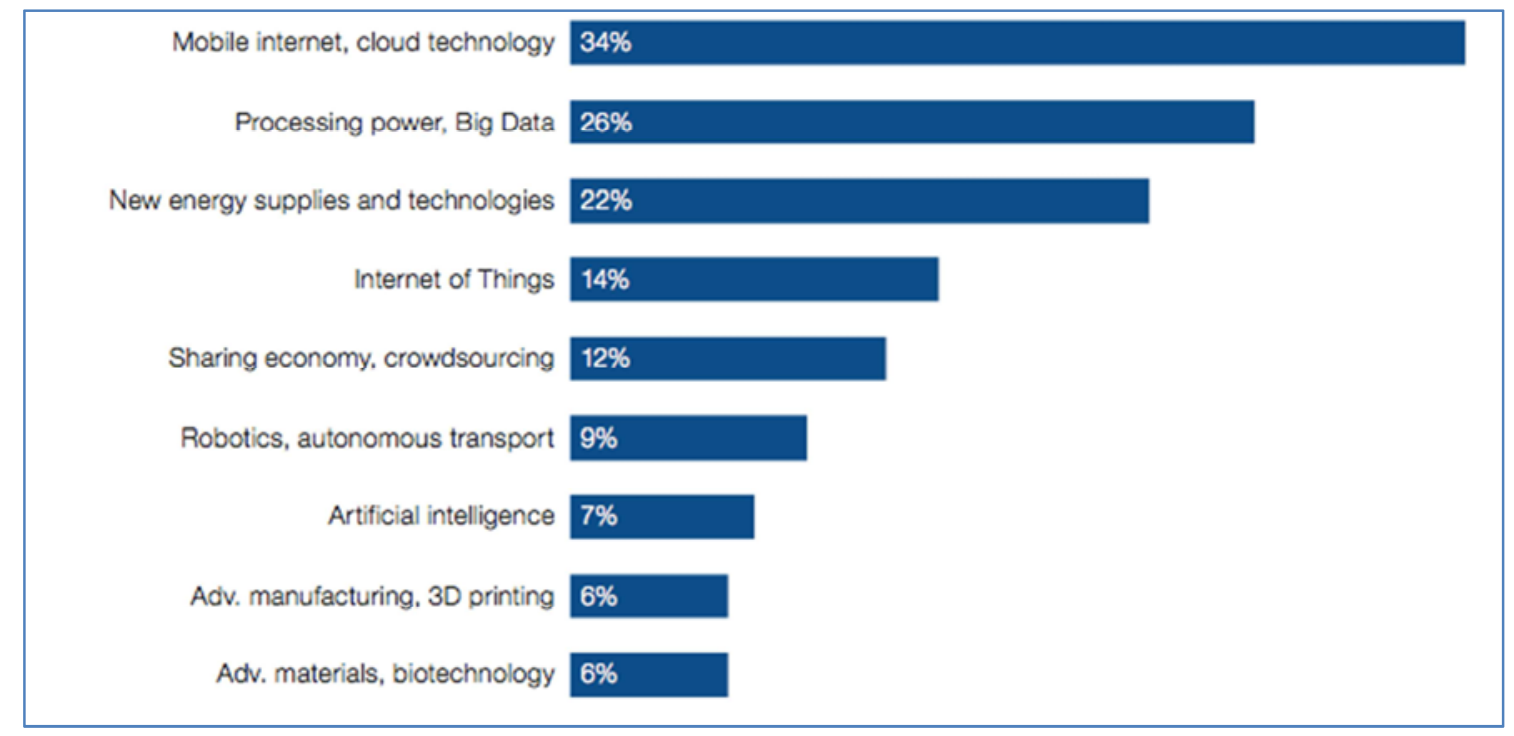

Figure 1. Future of job survey. Source: World Economic Forum, Davos, 2016.

The purpose of this work was to shape a new dimension of strategic marketing in the coming decades with the help of advanced technologies incorporated within the human body. This research is a very new futuristic approach, which tries to see beyond the borders of the ecommerce.

The study resulted from an office research, analyzing different sources of information in the field of new technologies. The present paper was initially aimed at sensitizing, both the marketing strategists and consumers, on the evolution to a new technology that will be incorporated within the human body. Authors have had this goal because they consider that the digitalization of the human body will most likely conduct to a radical transformation of marketing arena for a very simple reason: the digital consumer behavior will significantly change, being in a continuous run for information, products and services.

The ultimate goal of the research is to analyze the impact that embedded technology could have on the marketing environment and on customer behavior. Authors aimed to draw the attention that the actual marketing concepts appear to dismantle and to embrace new dimensions and meanings.

Electronic world from 1947 to new world of chips and biometrics

The electronics era began in 1947 , with the invention of transistor and silicon-based semiconductor technology. Seven decades later, electronic devices vital for our everyday lives surround us. Processing technologies and nanotechnology have rapidly led to their development and miniaturization. For many years, silicon was the only option for electronics, but current developments have brought new trends into attention to explore and develop in the coming years. The graphene discovery opened the way to 2-D electronics because of its high thermal and electrical conductivity, as well as the mechanical flexibility of its two-dimensional hexagonal mesh, thick of only one atom. Graphene is the most resistant material ever tested. In addition, maybe it started the 2-D revolution in the field of electronics, but allotropic thick atom forms of silicon, phosphorus and tin have the same honeycomb structure of graphene, but different properties that make them suitable for other applications. The fact is that all four have the potential to transform electronics, as we know it, making possible to miniaturize, increase performance and reduce the cost of devices. Already, companies like Samsung and Apple are developing graphene applications.

Another important leap was the development of conductive polymers, which brought in 2000 the Nobel Prize to Alan J. Heeger, Alan G. MacDiarmid and Hideki 
Shirakawa, who demonstrated that plastic could lead electricity [3]. This has led to organic electronic materials, made of carbon-based molecules or polymers using chemical synthesis. They do not yet compete with their conventional counterparts, but have their advantages, including reduced costs, mechanical flexibility, adaptability and biocompatibility, making organic electronics an optimal choice for certain applications.

Commercially available high-tech products that rely on organic semiconductors such as curved-screen TVs, smartphone screens, and portable solar cells already demonstrate their industrial maturation. This market expect to grow rapidly in the coming years.

In 1971, besides the resistor, capacitor and inductor, the electronics also proposed a fourth element of the circuit board - "Memristor" (memory + resistor). It was not until 2007 that Hewlett Packard's engineers discovered its potential, and since then, many researchers believe that electronics could go into a new era, that of "Ionics."

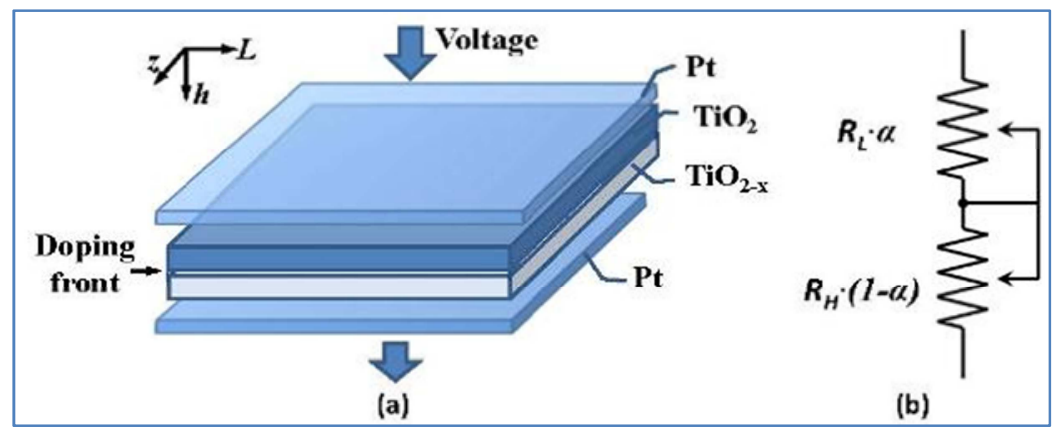

Figure 2. Memristor.

While conventional transistors operate using an electron stream, the memristor combines electrons with electronically charged ions or atoms. In transistors, once, the electron flow is interrupted (eg by extinguishing the system), all the information is lost. Instead, memristors "memorize" and store information about the task they crossed, even when the power is off.

That is why they are paving the way for a better memory of information and promise to provide faster, safer and more efficient storage devices. There will be no loss of information anymore, and we will be able to start or stop computers instantly. In recent years, HP has been working on a new type of computer system based on memristor technology by 2020 .

Somewhat in the same area of innovation is the "spintronic" - the use of the fundamental property of particles known as "electronic spin" for processing information. By transferring information through both the task and the spin of an electron, the devices could gain a greater diversity of functionality.

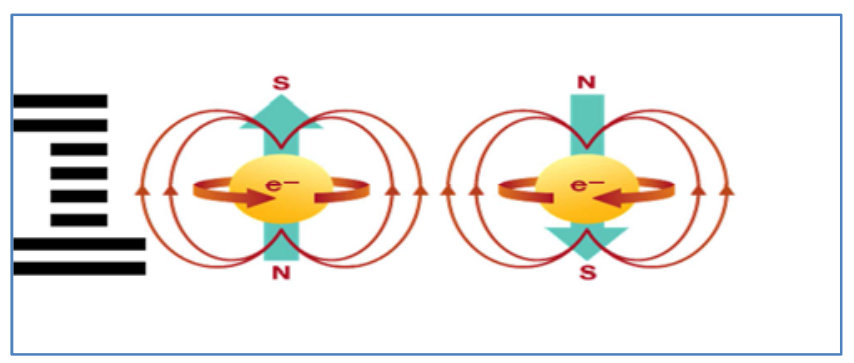

Figure 3. Spintronic.

Spintronic technology is in testing phase in data storage devices, such as hard drives, but it is promising about digital electronics in general: increased processing power, higher data transfer speeds and an increased capability of information storage. In addition, spintronic devices will miniaturize, more versatile and robust compared to their silicon-based "relatives".

By the way, miniaturization, this is the ultimate goal of electric circuits. A branch of nanotechnology, known as "mono-molecular electronics", uses individual molecules or groups of individual molecules as electronic "raw material". This type of approach will make the bulky material, even rough, of the current devices replaced by individual molecules. The smaller the dimensions of the electronic components, the lower the electricity consumption, and the sensitivity (and even the performance) of the device increases.

Monomolecular electronics could also lead to selfassembly, a phenomenon whereby the components of a system spontaneously merge with each other because of interaction or environment factors to form a larger functional unit. Several molecular electronics solutions are developed, including molecular wires and single molecule transistors, but this type of electronics is still in its infancy and none of these inventions has left the lab. When they do, the world will change forever.

Welcome to the brave new world of chips and biometrics

Bracelet projecting smart phones screen on arm.

French engineers from cicret. com are working on a bracelet CICRET to display intelligent mobile phones screen on the arm holder. Manufacturers say that Cicret bracelet, which is in the prototype stage, will make our skin our new tablet. They promise that we will be able to read emails, play your favorite games, answer calls, check the weather ....etc.... so, we can do whatever we want with our arm [4]. 


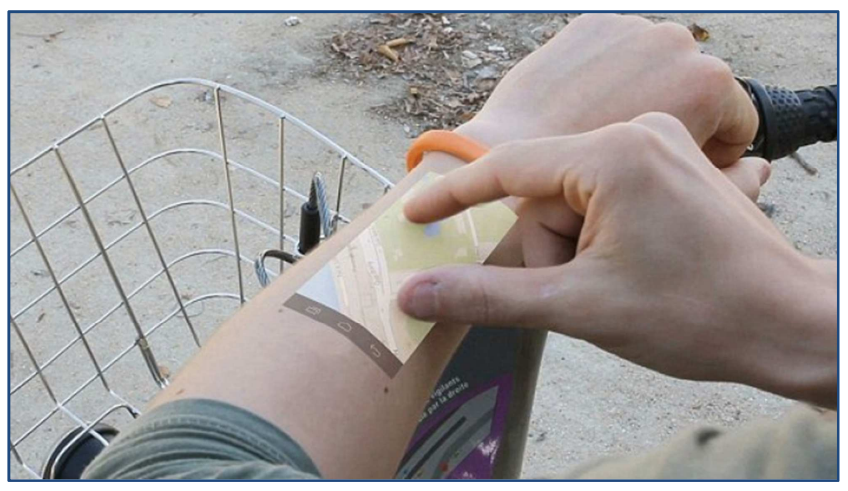

Figure 4. Smart bracelet. Source: dailymail.co.uk.co.uk

Cicret bracelet is equipped with a memory card, processor, accelerometer, micro-USB, and Wi-Fi and Bluetooth communication. The essential element of the bracket is socalled pico-projector, which is composed of a series of proximity sensors. Pico projector designs interface on arm. When the owner touches the screen with his finger, switch off one of the eight proximity sensors, which transmit the information at the processor within the bracelet.

French idea has attracted criticism because it seems that there is not yet a functioning prototype, nor too serious guarantees that it will be done. The inventors, however, say they need 700,000 euros to complete the prototype and another 300,000 euros to develop application bracelet. If they succeed, Cicret bracelet will be available in 10 colors and 2 sizes of drive -16 and 32 GB.

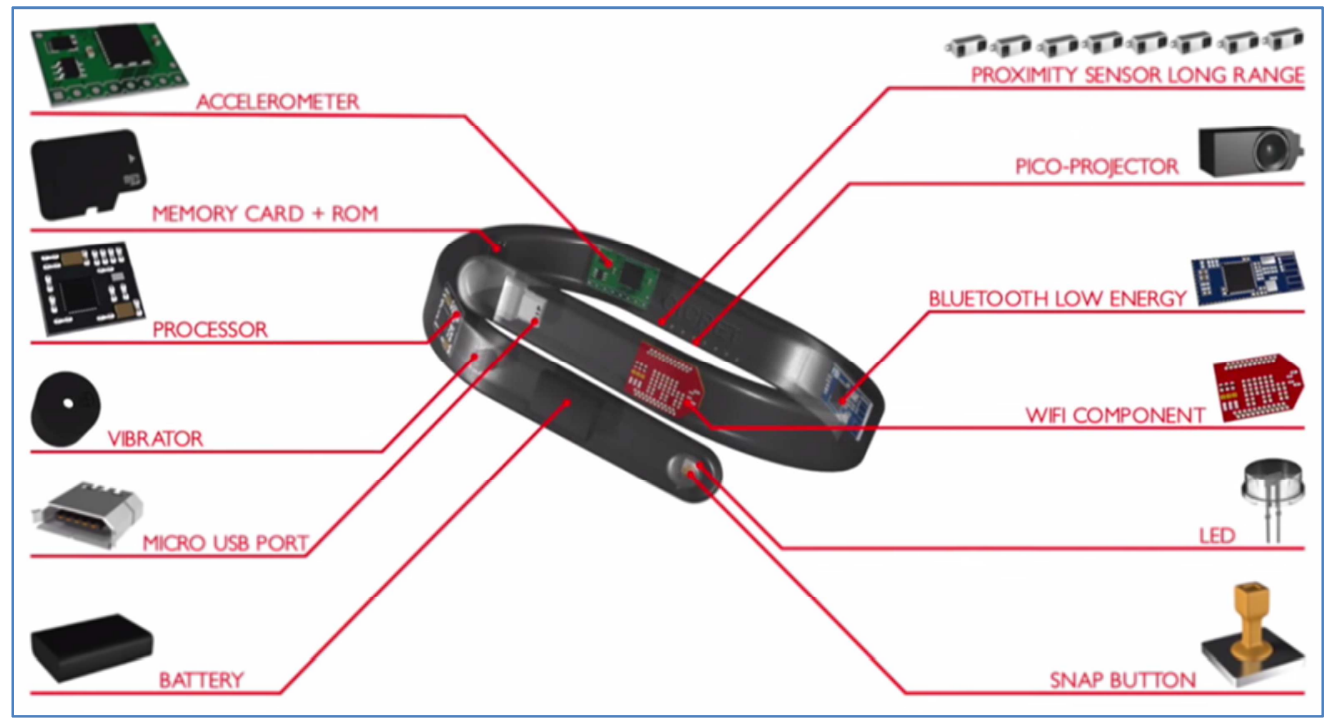

Figure 5. Smart bracelet connectivity. Source: www.cicret.com.

Electronic Tattoo capabilities, or rather bearer computer

In 2011, an international team of scientists announced a breakthrough: epidermal electronic system. It is a special electronic circuit, very thin, placed directly on the skin, designed to stretch, to twist and receive information from body movements.

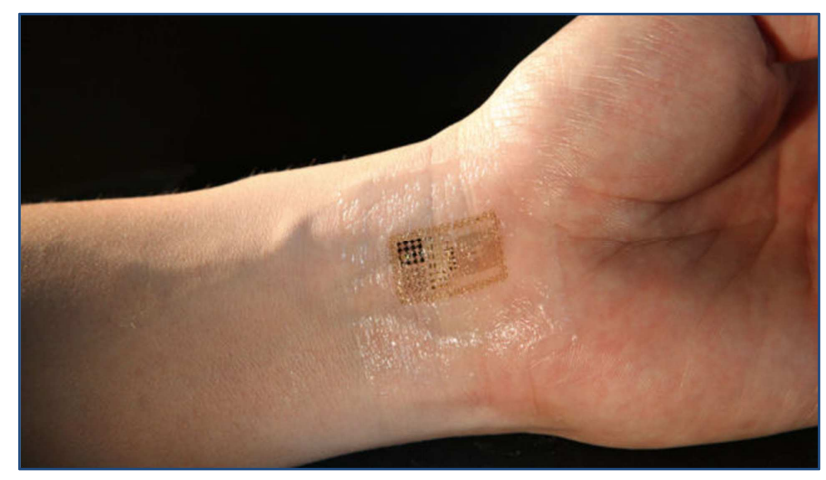

Figure 6. Electronic tatoo. Source: dailymail.co.uk.co.

Their discovery has made the transition from hard and rigid format of the traditional electronic devices to a structure softer, more dynamic. Specialists from different laboratories have developed special electronic circuits, whiskers, turned and mounted on a thin rubber substrate with elastic properties similar to skin. The result is an electronic patch that can bend, twist, stretch and contract without affecting performance and electronic.

One of the researchers involved, Sir John Rogers, professor of materials science and engineering at the University of Urbana-Champaign in Illinois, who worked for more than a decade to improve worn on the skin or implanted flexible devices, said the most promising applications the new technologies are in medicine. These are replacing conventional electrodes, which require supports, wire and adhesive gels. The system should have the ability: to monitor electrocardiogram, electromyogram, electroencephalogram; to stimulate muscle tissue, being used for physical rehabilitation; to monitor sleep; to serve as a means of communication for people with speech difficulties etc.

In addition, according to Washington Post 2016, in the near future, hospitals and doctors' offices may equip patients with temporary electronic tattoos. These small patches have a lot of information in a small space and can help reduce medical errors while improving care [5]. More patches models developed by the team of John Rogers entered or are 
about to enter clinical trials in hospitals in the US and Europe and expects the first commercial models to become available later this year, 2017.

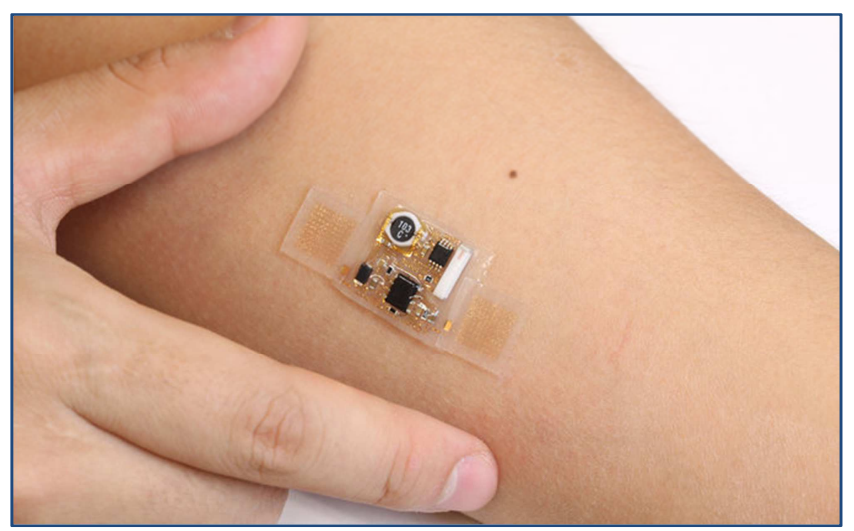

Figure 7. Bearer computer. Source: dailymail.co.uk.co.

Computer bearer supporters (photo) are extremely excited about the endless possibilities in terms of style. "It turns out that the mechanics of these devices is $100 \%$ compatible with standard temporary tattoos that children use," says Rogers. They look like tattoos, and each part contains different sensors, suitable to the aim pursued. Scott Pomerantz, director of the company that developed the electronic tattoos, MC10, Cambridge, Massachusetts, states: "Our devices are not like today bearer devices that are worn by people for a period and then thrown in a drawer. Ours are all over you. We have the smallest, most flexible and elastic bearer computer and you can reap all kinds of biometric data related to your move" [6].

Rogers' team is not the only working to produce electronic devices to skin. Takao Someya leads a group of scientists from the University of Tokyo working on developing electronic skin, made from organic semiconductors and carbon nanotubes. It looks like a sheet of flexible plastic and elastic containing many sensors and placed on real skin. Japanese strive to add a layer of LEDs to turn it into a functional screen placed on the skin.

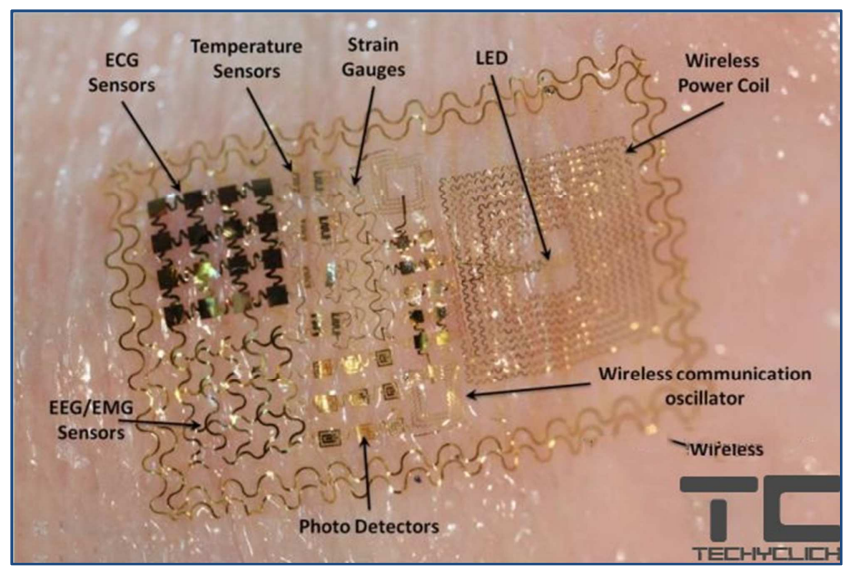

Figure 8. Electronic skin. Source: dailymail.co.uk.co.

Zhenan Bao from Stanford University is working with organic semiconductors to develop an electronic film could be as sensitive as human skin and could be applied to robotic limbs. Additionally, researchers from the University of California are working to produce special inks that could allow scientists to draw directly sensors on the skin.

Subcutaneous devices - merger of biological and electronic world

Statements of inventors on the possible merger of biological and electronic world we give thoughts to come. Engineer Todd Coleman, who also participated in epidermal electronic system design, said: "This technology offers a huge conceptual breakthrough in fusion of the biological and the electronic world in a very natural way. Somehow, the border between electronics and biological world is becoming vague" [7].

Referring to their future intentions, John Rogers said:, I would like to extend the functionality of such devices not only fit seamlessly into the human body in a mechanical sense, but also to communicate and interact with tissue in ways which go beyond the electrons and photons, the fluids and biomolecules.

Currently, progress and inventions occur with astonishing speed, in part, because man no longer satisfied with progress today, but always wants more and more sensational. Today, the greatest bearer devices are on the wrist. Nevertheless, everyone anxiously could ask: what next? New Deal Design Company, who designed the bearer line Fitbit devices, is now working on a new project called under-skin. It is a smart digital tattoo implanted subcutaneously in hand and will interact with any electronic device touched the person. Operating by electrochemical energy of the body, the device subcutaneously will be always on, can send signals to unlock the door when a person touches the doorknob of the door, to exchange data with another person only by a handshake, to provide information of a medical nature (pressure, blood sugar, temperature) or to ensure that the credit card operated only when in the hands of the owner.

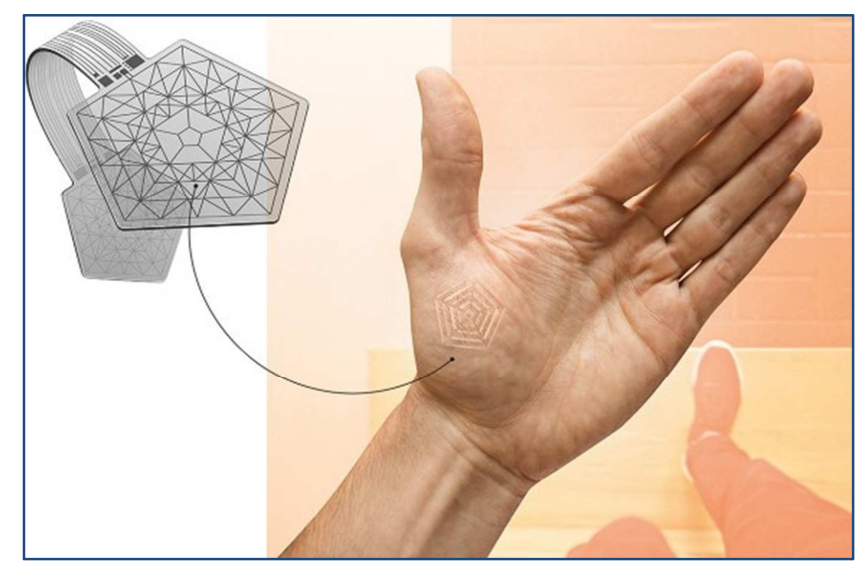

Figure 9. Subcutaneous devices. Source: dailymail.co.uk.co.

In addition, the device can provide information about the owner in two ways, depending on the degree of confidentiality required: can present an image knuckle or a larger screen existing discreetly hand. In addition, it can take 
account of gestures made by the owner, catching shade communication between him and another person.

Jaeha Yoo, director of design company New Deal Design stated in Daily Mail that "...even though there are lots of cultural boundaries regarding the idea of implanting something within the body, there are tattoos and piercings implanted within people body starting from birth. These things are happening now." [8].

New Deal Design Company is confident that it could build the device subcutaneously in the next five years, given the current state of electronic research. The most difficult work will require flexible screen rather than the sensor, communication, implant or using body energy. Gadi Amit says even that we will see soon devices bearing subcutaneous because biology, medicine, electronics and cultural wisdom will weave lots of interaction [9].

Computers, which are dissolving

Another idea of these scientists was to fabricate ultra-thin electronic devices which, having fulfilled their mission, dissolve in the body. This type of electronic dissolves in a controlled manner are composed of silicon oxide and magnesium oxide in a protective layer made of silk. The silicon dissolves in water, but in a very long time. As a result, researchers have used extremely thin silicon sheets, called Nano-membranes, which dissolved in days or weeks.

Smart tattoo authentication

Another use for smart tattoos is authentication for phones and tablets. Regina Dugan, head of special projects of the company Motorola Mobility and former project leader Agency of Advanced Research Projects [Minister of Defense] says: "We think a variety of authentication options. It can start from things like coupons or trinkets, but think of the means of authentication worn on the skin for a week. We are talking about an electronic tattoo" [10].

Placed on the wrist or arm has "sensors and an antenna that can recognize your devices then send a signal authentication". According to her: "Teens may not want to wear a watch, but you can be sure they wear a tattoo just to defy parents". In an article in the Daily Mail is noted: "MC10 originally designed tattoos, called bio-stamps to help medical teams to assess patients' health or remotely, or without the need for large and expensive equipment. Motorola says that circuits, which contain embedded antennas and sensors, could be adapted to work with mobile phones and tablets. Then, mobile devices confirm the identity of the owner and enter the account automatically. This could prevent thieves and others to access the phone application, etc. if it is lost or stolen."

Another Motorola authentication idea is Motorola Pill, also called vitamin identification, which is a pill that emits a signal authentication on 18 bits, which can connect the person to devices. "Vitamin" containing a chip, swallowed and once arrived in the stomach, gastric acid serves to the position of electrolyte and activate the chip. Regina Dugan present this technology as a real magic: "That means it will be my first superpower. I really want this superpower, that means my hands are like threads, and when I reach the phone, computer, door, car, are directly recognized" [11].

\section{Results}

Human body digitalization - consequences in marketing arena in the coming decades.

Digitalization is already part of corporate life and bring not only new business models, but also efficiency in business processes, from the production and operational departments, to the marketing and sales.

Human body digitalization process will profoundly change marketing strategies in the coming decades, in that it will make the transition from social networks (Facebook, Twitter...etc.) and data mining to the capability of marketing strategy to "collect" information about each client in real time, because then it can help marketing strategists to make the right decision in order to meet the established objectives. If Facebook is a reality faced almost the entire planet daily, Data mining is the process analytical exploration of large databases - Big Data - for searching and finding patterns consistent and / or relationships systematically between different variables, followed by applying the detected patterns of the new sets of data.

Authors consider that one of the most surprising predictions that can make marketing strategists, using human body digitalization, is about shopping. Retailers will be able to combine digital products and services with storefronts, meaning that potential customers can in physical or virtual touch and try the clothes before they order, and will arrive in minutes.

In the next decades, or even sooner, marketers will be able to have information in real time, from billions of events in which are attending potentials clients. Through digitalization of human body technologies, marketers will learn to understand people's needs, their likes, emotional reactions to a product/service, thus using this information help them to develop new marketing strategies.

Companies to expand market of their products and develop new ones can use technologies such as digitalization human body, a kind of biofeedback. Authors expect who will know how to exploit the information offered by human body digitalization is de facto and de jure new "Lord of the Rings" of marketing arena.

Authors believe that beyond connecting devices implanted in the human body systems to IT systems, the real revolution in marketing brought by human body digitalization is the ability to detect, almost in real time, inherent qualities or existing relationships in big amounts of data ever-increasing volume, velocity and variety. Even some critical information, timely discovered and shared with the right person can optimize business processes, leading to more informed decisions, identify new revenue opportunities and predict the behavior of customers and partners in unimaginable ways before.

Human body digitalization project, the merger of biological and electronic world, is a paradigmatic leap and ontological change. Our fundamental notions of what it means to be human and what it means to be "humanity" base on subject-object dichotomy. Human body digitalization 
introduces a third dimension to the current existential relations: Big Data and a realistic scenario always present in any subject-object interaction.

On the same time, authors proactively prevent that could be negative consequences that we have to consider if these technologies are used by political parties, governments and other organizations to promote agendas / ideological interests.

If we relate to "the next epoch-making invention" of humankind, authors say that human body digitalization project is as important as food or knowledge - a paradigm shift, a disruptive event, creator of a new vision of our time.

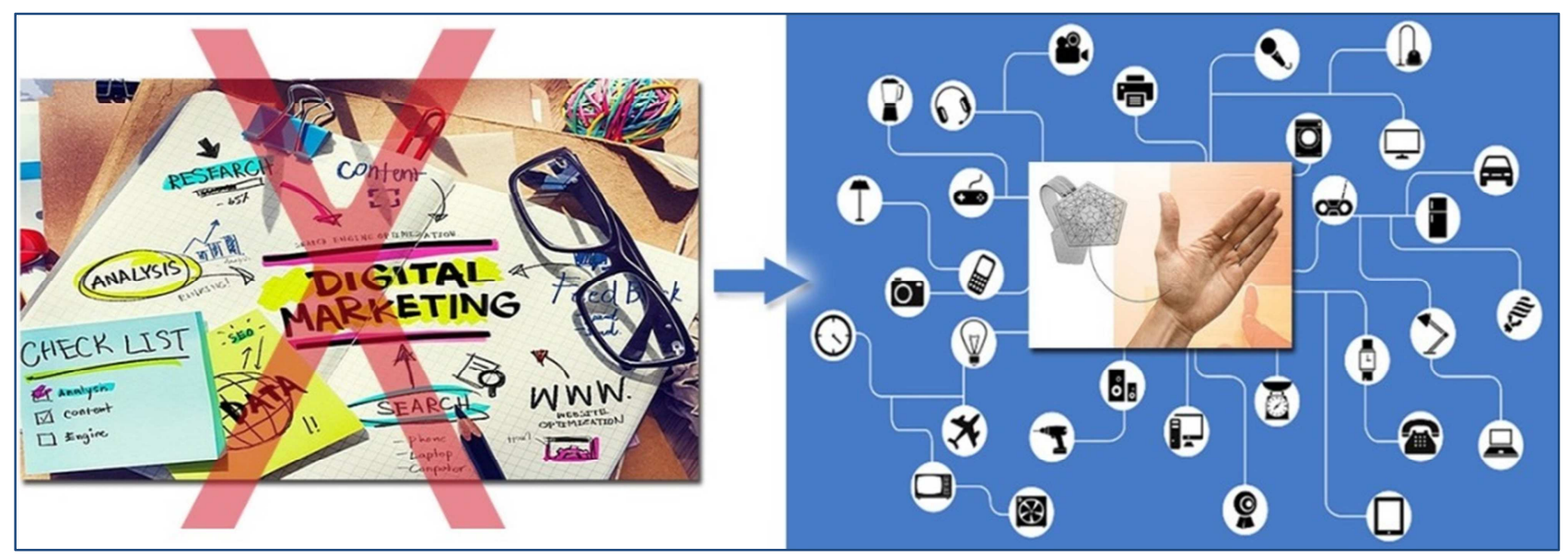

Figure 10. Shaping a new dimension of marketing.

\section{Discussions}

Implications for Business Marketing Practice.

Human Digitalization process will not be longer a novelty, but for many corporate executives must probably will become a zero priority for the radical change it can bring in a marketing arena.

From the visionary perspective of the authors, human body digitalization will have a crucial impact on both, horizontally and vertically, business and marketing of services and products. This implies that, on the one hand, business companies need to better integrate and digitize their vertical data flow, from product development and procurement to processing and transport logistics, and on the other hand, it involves a horizontal collaboration with key suppliers, customers and other partners, for example by using product identification and monitoring solutions.

Human body digitalization implies for companies the creation in advance of complex digital solutions for the development of new products and services, covering the entire life cycle of the product and thus facilitating close contact with end-users. Companies will give up their current marketing approach and invest in digital services to create complete solutions tailored to their customers' ecosystems.

Analyzing the data provided by the technological implants in the human body will provide a valuable insight into the management and marketing of companies on how the products are used and make possible the long-term relationship with customers. Data analysis tools allow for product development and enable companies to expand their services and better align their bids with the needs of clients.

Digital eco-systems, as a new marketing system between companies and customers, can only work if all participants can trust their data will not reach the wrong hands. This requires considerable effort on the part of companies, substantial investment in system security and clear data protection standards.

Research is limited to the fact that any innovation, as is the case with the implementation of technology in the human body, cannot have only benefits. Human Digitalization process creates benefit for business companies in terms of time and cost, but does not compensate by creating the right jobs.

The authors believe human body digitalization process will conduct to unnecessary functions from today's marketing, functions, accomplished today by people with medium or even higher education. The phenomenon is not new, let us think about how many cashiers have canceled by introducing ATMs, e-banking applications etc... Marketing unemployment will become a universal problem that states will have to deal with.

In addition, the authors foresee major changes in the educational system, where the strong need for marketing specialization diplomas will most likely disappear and will emphasize the need for a high-quality education, visionary applied. Universities living today from stamped marketing diplomas will most likely disappear. Learning will become a continuous process, and those who lose the step will lose access to this privileged category.

The implementation of such technology could be perceived as an Achilles heel. The authors of this study raise two vulnerabilities/limitations here for subsequent discussions: cyber security and protecting our privacy.

The evolution of marketing remains, however, an important educational need and, why not, a source of inspiration to find new solutions in the future.

Concluding, the problems that companies will have in the face of human body digitalization will be those related to the 
lack of a culture, vision or internal training in the digital domain.

\section{Conclusion}

Human body digitalization is a new and challenged theme at the same time on the marketing arena. The authors advice marketers to adapt with the development of the technology embedded in the human body and remodel their strategy into a consubstantial reality: physical and virtual. The authors argue that, in the future decades, the purchasing decision will be a major part of human digital entities based on established algorithms depending on their needs.

According to the authors, the new embedded technology will gradually lead to minimizing the importance to the disappearing of following marketing methods currently used as competitive \& business intelligence, marketing consultants, pay per click promotion on platforms like AdWords, Facebook Ads or LinkedIn Ads, social networking, E-commerce sites in the top of online sales channels, media buying campaigns through the most popular blogs and online publications, affiliate marketing through different affiliate platforms.

From authors perspective is clear that revolutions that take place in biotechnology and artificial intelligence, which redefine what means to be human, pushing more and more the boundaries of our physical and mental capacities, will force us to reconsider our moral and ethical limits. If all this progress will do us good or bad, it depends only on us and how mature the human species really is.

\section{References}

[1] Popescu, F. \& Scarlat, Cz. (2017). Human Digital Immortality: Where Human Old Dreams and New Technologies Meet. IGI Global, Pennsylvania (USA).

[2] World Economic Forum (2016).

[3] The Royal Swedish Academy of Sciences (2000). The Nobel Prize in Chemistry, 2000: Conductive polymers.

[4] Your skin is your new touchscreen. Retrieved on the $10^{\text {th }}$ of May 2017, from www.cicret.com.

[5] The Washington Post (2016). This electronic tattoo turns your skin into a screen.

[6] Pomerantz, S. (2016). Ultra-thin, Tattoo-like Wearables Enabled by New Technology Partnership.

[7] IO 9 (2011) - Breakthrough: Electronic circuits integrated with your skin.

[8] Daily Mail Online (2014). Is this interactive tattoo the future of 'wearable' technology?

[9] Fitbit (2016) - The Most Worn Wearables.

[10] Dugan, R. (2013). Wall Street Journal.

[11] Dugan, R. (2013). Apple, Motorola Look to New Authentication Methods. 